\title{
95 Excessive sound stimulation of the ear
}

A May cause transitory residual masking.

B A long lasting temporary threshold shift (TTS) recovers within $16 \mathrm{~h}$.

C Can produce an acoustic notch at $6000 \mathrm{~Hz}$.

D A short, intense single noise exposure may lead to permanent perceptive deafness.

E Can evoke the Tullio phenomenon only with an intact and mobile middle ear mechanism.

\section{Infrasound}

A Is easily detected by the human ear.

B Occurs in high speed automobiles particularly if the windows are open.

C May induce nystagmus.

D Could have caused the destruction of the 'Walls of Jericho'.

E Can be recorded on a normal tape-recorder.

\section{Hearing conservation programmes in industry}

A A sound profile of the potentially hazardous area is essential.

B Temporary threshold shifts do not adversely affect audiometric testing.

C The most effective hearing protector is the ear muff.

D Hearing protection need not be worn continuously in a high risk zone.

E Observation booths may be a practical solution. 